Meta

Journal des traducteurs

Translators' Journal

\title{
La terminologie de la texture des aliments
}

\section{Christine Daniel et Alain-Claude Roudot}

Volume 52, numéro 2, juin 2007

URI : https://id.erudit.org/iderudit/016075ar

DOI : https://doi.org/10.7202/016075ar

Aller au sommaire du numéro

\section{Éditeur(s)}

Les Presses de l'Université de Montréal

ISSN

0026-0452 (imprimé)

1492-1421 (numérique)

Découvrir la revue

Citer cet article

Daniel, C. \& Roudot, A.-C. (2007). La terminologie de la texture des aliments. Meta, 52(2), 342-351. https://doi.org/10.7202/016075ar

\section{Résumé de l'article}

En sciences alimentaires, la normalisation terminologique se heurte à un obstacle difficilement surmontable lié au caractère sensoriel des mesures. On utilise des termes qui, bien que paraissant très bien définis, recouvrent des concepts flous. À ce problème s'ajoute celui créé par l'existence d'une seconde méthode de mesure à base instrumentale. Les termes traditionnels usités sont très fortement chargés culturellement, difficilement utilisables avec la méthode instrumentale, et constituent un verrou important à tout développement scientifique. Nous proposons donc une terminologie à deux niveaux dont le premier est largement basé sur la terminologie sensorielle actuelle et le second précise chaque terme du premier niveau en fonction des phénomènes physiques concernés, ce qui permettrait de lever certaines ambiguïtés conceptuelles souvent gênantes.
Ce document est protégé par la loi sur le droit d'auteur. L'utilisation des services d'Érudit (y compris la reproduction) est assujettie à sa politique d'utilisation que vous pouvez consulter en ligne.

https://apropos.erudit.org/fr/usagers/politique-dutilisation/ 


\title{
La terminologie de la texture des aliments
}

\author{
CHRISTINE DANIEL \\ Université de Bretagne Occidentale, Brest, France
}

\author{
ALAIN-CLAUDE ROUDOT \\ ESMISAB, Technopole Brest-Iroise, Plouzané, France \\ alain-claude.roudot@univ-brest.fr
}

\begin{abstract}
RÉSUMÉ
En sciences alimentaires, la normalisation terminologique se heurte à un obstacle difficilement surmontable lié au caractère sensoriel des mesures. On utilise des termes qui, bien que paraissant très bien définis, recouvrent des concepts flous. À ce problème s'ajoute celui créé par l'existence d'une seconde méthode de mesure à base instrumentale. Les termes traditionnels usités sont très fortement chargés culturellement, difficilement utilisables avec la méthode instrumentale, et constituent un verrou important à tout développement scientifique. Nous proposons donc une terminologie à deux niveaux dont le premier est largement basé sur la terminologie sensorielle actuelle et le second précise chaque terme du premier niveau en fonction des phénomènes physiques concernés, ce qui permettrait de lever certaines ambiguïtés conceptuelles souvent gênantes.
\end{abstract}

\begin{abstract}
In food science, the main obstacle to terminological standardization is the sensory aspect of the measurements. Although terms may seem well-defined, they always concern very fuzzy concepts. This problem is increased by the fact that two different methods of analysis are concerned: sensory analysis on the one hand, mechanical analysis on the other. The terms we commonly use are imbued with cultural meanings, and are consequently difficult to use with mechanical methods, which are more objective. Terminological problems thus appear to be a major obstacle to any significant progress in this research area. In this paper, we propose a two-level terminology: the first level is based on classical sensory terminology, while the second gives a more accurate description of the physical phenomena involved in the sensation. We hope this two-level terminology will help prevent some conceptual ambiguities which have often restrained scientific development in this area.
\end{abstract}

\section{MOTS-CLÉS/KEYWORDS}

inter-compréhension, langue de spécialité, normalisation, sciences sensorielles, texture des aliments

L'analyse de texture des aliments est un domaine scientifique qui a débuté il y a un siècle environ, mais qui a réellement pris son essor au début des années 1960, notamment sous l'impulsion de R. H. Scott Blair et A. S. Szczesniak. Dès la fondation de ce domaine, le problème terminologique s'est posé en particulier pour définir le terme texture dans le domaine alimentaire. Ainsi, Smith (1947) la définit comme «the rigi- 
dity of solid units of food», quand Hall et Fryer (1953) donnent la définition suivante: «the term texture has come to mean how hard or how soft as well as how large or how small the individual kernels in the mass of food actually are». Des définitions plus sensorielles et plus limitées dans leur domaine d'application sont également proposées comme «the texture of cooked meat is the feel of smoothness or fineness of the muscle tissue in the mouth» (Ball et al. 1957). Finalement, un certain consensus est apparu autour de la définition donnée par A. Szczesniak (1998): «texture is the sensory and functional manifestation of the structural and mechanical properties of foods, detected through the senses of vision, hearing, touch and kinesthetics ». Cette dernière définition ayant le mérite de réconcilier les deux grandes catégories de praticiens tournés soit vers l'analyse sensorielle, soit vers l'analyse instrumentale de cette caractéristique organoleptique. Malheureusement, le consensus terminologique s'est arrêté à ce stade malgré quelques rares tentatives de remise en cause ou de redéfinition des termes classiquement utilisés (Jowitt 1974; Delêtre et Roudot 2003).

\section{Traduire le sensoriel}

L'analyse de texture consiste donc à analyser un produit alimentaire du point de vue de la sensation ressentie lorsque ce produit est mis en bouche avant son ingestion. En fait, on étend traditionnellement cette caractéristique en amont, considérant que la vue du produit, sa tenue en main (ou sous la fourchette) sont également à prendre en compte. Tous les sens sont ainsi concernés: le toucher lors de la prise du produit et dans la mise en bouche, la vue lors du choix, l'ouïe lors de la mastication. Le goût et l'odorat sont théoriquement exclus, mais les études de psychorhéologie montrent que leur influence est impossible à éliminer dès lors que les tests se font dans le cadre de jury de dégustation (Bagot 1999). Un des premiers travaux de l'analyse de texture va consister à nommer ces sensations le plus objectivement possible, c'est-à-dire à interpréter un champ sensoriel par la parole. Cette traduction intersémiotique est probablement l'élément déterminant dans la qualité du résultat à venir, car d'elle va découler toute l'analyse ultérieure du produit testé. Cependant, si l'on peut considérer avec Sarrukai (2001a) que la science est essentiellement basée sur la traduction multisémiotique de la nature, dans le cadre d'une analyse organoleptique se pose le problème supplémentaire de l'objectivité de la réponse reçue et de sa reproductibilité inhérentes au caractère sensoriel du problème posé.

\section{L'alimentation, un élément culturellement chargé}

L'homme n'a pas attendu que la science analyse son alimentation pour s'y intéresser. Depuis longtemps, l'homme parle de ce qu'il mange et qualifie son alimentation. Ceci est tellement vrai que ses goûts alimentaires font partie intégrante de sa culture: telle région apprécie plutôt l'huile d'olive, quand telle autre préfère les produits craquants ou gluants, etc. Ainsi, chaque culture a développé une terminologie adaptée aux produits qu'elle utilise. Cette terminologie est généralement floue du fait de recouvrements géographiques ou sensoriels, mais elle est amplement suffisante dans la vie quotidienne. Ainsi, lorsque l'on parle de fermeté pour une pastèque, traditionnellement on lui imprime une secousse brutale qui la fait vibrer, et c'est l'évaluation de cette vibration qui permet de qualifier la fermeté. Par contre, la fermeté d'un abricot 
sera évaluée en le serrant légèrement entre deux doigts et c'est la force de compression qui prendra le nom de fermeté. Le même terme est donc utilisé pour deux sensations différentes. On peut également trouver le phénomène inverse où la même caractéristique peut se retrouver sous plusieurs termes comme la tenue, la consistance ou le corps d'un produit pâteux. Ces caractères inhérents à toute langue de communication ne posent aucun problème dans la vie quotidienne où le contexte permet aisément de comprendre l'idée développée. Par contre, ce vocabulaire, très couramment pratiqué, est le vocabulaire qui est utilisé par défaut, faute de connaissance de vocabulaire plus précis, lors de toute caractérisation d'aliment. En effet, comme dans tout nouveau domaine en cours d'élaboration, la langue utilisée est la langue quotidienne des chercheurs, la langue idéale adaptée à ce nouveau domaine étant inexistante. La traduction scientifique de la texture réelle d'un produit sera alors floue, peu fiable et faiblement reproductible. La conséquence immédiate, qui a été tirée dès le début des années 1960, a été de préciser la terminologie afin de permettre une caractérisation la plus fine possible des textures alimentaires (Szczesniak 1963).

\section{Nécessité d'une langue spécialisée}

Ainsi que le rappelle $\mathrm{H}$. This (2002) en citant Lavoisier: "L'impossibilité d'isoler la nomenclature de la science, et la science de la nomenclature, tient à ce que toute science physique est nécessairement fondée sur trois choses: la série des faits qui constituent la science, les idées qui les rappellent, les mots qui les expriment. Comme ce sont les mots qui conservent les idées, et qui les transmettent, il en résulte qu'on ne peut perfectionner les langues sans perfectionner la science, ni la science sans le langage.» Appliquant ces idées, Lavoisier réforma la terminologie chimique (envers et contre tous) et ouvrit ainsi la porte au formidable développement de cette science au XIX ${ }^{\mathrm{e}}$ siècle. Cette remarque de Lavoisier, loin d'être une découverte, est appliquée de fait dans tous les domaines de la vie, que ce soit en navigation, en cuisine, en mathématique, en agriculture, etc. Les pratiques utilisent un vocabulaire spécifique considéré comme un jargon par les non-initiés, mais nécessaire à la compréhension et au fonctionnement du domaine considéré. Il ne s'agit donc pas de créer une nouvelle langue idéale au sens développé par Eco (1997), mais comme le suggère Sarrukai (2001b) d'aménager une terminologie spécifique au sein de la langue naturelle.

Dans le domaine qui nous concerne ici, cette nécessité ne fait déjà plus consensus, car certains chercheurs pensent que du fait de la forte prégnance de la culture de l'individu sur l'appréciation de l'aliment (et donc de sa texture), il est nécessaire de conserver un glossaire peu défini afin de permettre l'expression aisée de toutes les sensations ressenties par l'expérimentateur avec ses propres termes. En effet, la traduction de sensations sous forme verbale est suffisamment difficile pour ne pas rajouter, de surcroît, l'obligation d'utiliser un vocabulaire spécifique précis et strictement défini qui ne sera jamais en parfaite concordance avec la sensation ressentie. L'être humain est vivant, de même que ces sensations, et il est impossible de les classifier de manière stricte, donc il faut conserver les terminologies individuelles, et ne rien leur substituer.

Cependant, cette position n'est pas tenable pour deux raisons principales: la première est la nécessité dans tout domaine scientifique de pouvoir communiquer précisément ses résultats, ce qui n'est envisageable que par l'utilisation d'un mode de 
communication commun, et donc d'une terminologie commune. Le fait que la texture est un phénomène très lié à des caractéristiques sensorielles ne remet pas en cause ce fait, l'exemple du développement de la dégustation des liquides, et particulièrement des vins, est là pour s'en convaincre. Les résultats acquis dans ce domaine sont tout à fait conséquents, et sont à rapprocher de la redéfinition terminologique fine des termes classiquement utilisés en dégustation des liquides (Coutier 1994; Mellor 2002). Ces résultats ont d'ailleurs été repris dans le cadre des dégustations des fromages et du café, par exemple (Bérodier et al. 1997). La deuxième raison impliquant la création d'un glossaire précis et spécialisé provient du fait que l'analyse de texture n'est pas uniquement un domaine où le sensoriel est roi, mais également un domaine dans lequel se développe une approche instrumentale de plus en plus importante, liée notamment à l'importance de la texture dans les transformations technologiques des aliments. Les caractéristiques organoleptiques n'étant plus évaluées de manière sensorielle, il est inadéquat, voire impossible d'utiliser une terminologie strictement sensorielle pour exprimer ses résultats. Or les deux méthodes, sensorielle et instrumentale, doivent pouvoir se rencontrer et être comparées, car, au final, ce sont les mêmes éléments qui sont évalués, par des méthodes que l'on qualifie souvent de subjectives (analyse sensorielle) et d'objectives (analyse instrumentale) (Szczesniak 1963).

\section{Exemple de traduction sensorielle: la notion de croustillance}

Au cours des dix dernières années, la croustillance est devenue une notion emblématique de la texture des aliments et, dans le même temps, l'exemple de la difficulté d'expression des caractéristiques sensorielles:

Crispness appears to be the most versatile single texture parameter. It is particularly good as an appetizer and as a stimulant to active eating. It is notable as a relaxing or satiable texture. It appears to be universally liked and is often used as a popular accentcontributing or dramatizing characteristic. Crispness is very prominent in texture combinations that mark excellent cooking and is nearly synonymous with freshness and wholesomeness (Szczesniak 1988).

En outre, avec le développement du grignotage (passage de trois repas classiques par jour à l'ingestion quasi continue de nourriture tout au long de la journée, sous forme de biscuits, plaquettes de céréales enrobées de chocolat, céréales soufflées, etc.), la texture la plus recherchée est devenue la texture croustillante, ou ses voisines les plus proches: les textures craquantes, croquantes et fragiles. On retrouve le même phénomène dans les pays de langue anglaise avec les notions crispy, crunchy et crackly. C'est d'ailleurs surtout en anglais que le problème terminologique a été soulevé. Si tout le monde est d'accord sur le phénomène de rupture brutale du produit croustillant (crisp), les conditions précises sont très variables, certains y rajoutent un bruit de haute fréquence, d'autres une destruction complète, d'autres encore, un émiettement. Dans certains cas, ce caractère se révèle au premier croc, mais parfois il intervient tout au long de la mastication. Des incohérences apparaissent également chez les mêmes expérimentateurs lorsqu'ils décrivent le terme crisp (présente une fracture nette, c'està-dire une cassure nette associée à un son aigu) et lorsqu'ils analysent la sensation associée (l'analyse statistique des résultats de leurs tests expérimentaux montre une opposition entre les termes crisp et cassure nette). On propose également le céleri comme le produit (parmi ceux proposés) présentant la texture croustillante la plus 
marquée mais il ne présente pas de cassure nette (cités par Daniel 2003). La différence sensorielle entre croustillant et croquant est également très difficile à repérer, puisque les deux présentent les mêmes caractéristiques, la différence provenant parfois d'une rapidité de rupture supérieure pour le crisp ou, ce qui peut éventuellement s'en rapprocher, par une fréquence acoustique légèrement supérieure. Cependant, nombre de produits sont décrits avec les deux textures simultanées (Szczesniak 1988). Les études systématiques de la texture des aliments ont montré que le terme crisp peut être adopté dans deux cas tout à fait différents: sur des produits secs (biscuits) et sur des produits très aqueux (fruits et légumes). Ceci a conduit à un découpage de cette notion en $d r y$-crisp et wet-crisp (Jowitt 1974), mais certains préférent réserver le crisp aux produits secs, et associent le wet-crisp au crunchy (Fillion et Kilcast 2002), Jowitt se déclarant, quant à lui, favorable à la conservation de wet-crisp et à l'utilisation plutôt du terme brittle pour dry-crisp.

On s'aperçoit très clairement que si la notion initiale est considérée comme importante, le consensus sur les termes à utiliser est loin d'être atteint, de même que le consensus sur la signification de ces termes. Ces difficultés peuvent être rapprochées de l'appréciation de R. Barthes (1970) sur cette notion: "Qui a une vertu presque magique, un certain pouvoir de réveil, de stridence, opposé au caractère liant, lénifiant des nourritures sucrées.» Comment décrire scientifiquement un produit qui a un comportement magique?

Un autre problème qui apparaît très rapidement est la compréhension interlangue. En effet, les recherches sur la texture sont très internationalisées et il est donc nécessaire d'obtenir des traductions précises des termes utilisés. Or, la forte prégnance de la culture, signalée plus haut, entraîne de grandes variations entre les terminologies des différentes langues naturelles ainsi qu'entre les utilisations relatives des notions (Rodriguez et al. 1997). On remarque ainsi que si en anglais, ou en français, les termes concernant la texture croustillante sont en faible nombre (environ 3 ou 4), ce n'est pas du tout le cas en japonais ou en chinois, où l'on en a dénombré une vingtaine (Daniel 2003; Szczesniak 1988). Les différents dictionnaires proposés sont alors incapables de proposer des termes convenables en ne se basant que sur des traductions de termes (Drake 1989; Lawless et al. 1997). De surcroît, le recouvrement entre deux termes supposés équivalents dans deux langues différentes, n'est jamais parfait. Ainsi, Daniel (2003) signale l'utilisation du terme crisp sur la laitue aux États Unis alors que sa traduction classique 'croustillante' n'est jamais utilisée en Français pour ce type de produit, où on lui préfère le terme 'croquante' traduction de crunchy. S'agit-il d'une différence sensorielle, culturelle ou terminologique? La question n'a pas été tranchée à ce jour. Andani et al. (2001) montrent le même phénomène de recouvrement partiel dans le cas de l'expression de la farinosité de la pomme, entre cinq langues européennes.

\section{Quelle base pour une nouvelle terminologie?}

S’il peut désormais paraître assez clair que la recherche en texture des aliments nécessite au moins un toilettage de la terminologie utilisée, et plus probablement une mise à plat de cette dernière, plusieurs méthodes entrent en concurrence dans chaque cas avec leurs avantages, inconvénients et partisans (Daniel et Roudot 2003). On peut aisément remarquer que le fait d'être un domaine scientifique à deux 
aspects complémentaires, sensoriel et instrumental, est la cause principale de ce choix de possibilités.

\section{Les méthodes à base sensorielle}

L'analyse sensorielle des propriétés organoleptiques des aliments est beaucoup plus ancienne que l'analyse instrumentale et, sans se poser strictement le problème de la terminologie à utiliser, les chercheurs de ce secteur ont rapidement été amenés à tenter de résoudre le problème: comment transformer les termes de la langue naturelle en termes scientifiques, c'est-à-dire tendant vers la monosémie, permettant l'identification et la différentiation des caractéristiques sensorielles (Civille et Lawless 1986).

La première solution qui a été utilisée consistait à proposer une liste de différents termes de qualification de texture à un jury de dégustation. Chaque membre de ce jury doit rapprocher chaque terme du produit alimentaire qui lui paraît le plus caractéristique. Par exemple, pour le terme anglais soft, les produits classiquement cités sont la pomme de terre et la banane (Szczesniak et Skinner 1973). Le promoteur du test sensoriel compare ensuite les résultats et décide d'assimiler le terme de texture au produit le plus souvent indiqué. Cette méthode comporte de nombreux inconvénients, car elle n'est basée que sur l'idée que les dégustateurs ont des termes de classification, et le souvenir antérieur de dégustation de produits. Aucune définition n'est clairement exprimée, et les 'définitions' obtenues ne sont utilisables que lors du test en cours pour les dégustateurs choisis, car aucune mémoire n'est conservée des processus de choix. Cette méthode, bien que toujours utilisée, n'offre donc aucune espèce de solution au problème terminologique posé. Il s'agit pourtant de l'archétype des méthodes utilisées en analyse sensorielle, où, pour rechercher l'objectivité et la reproductibilité de l'expression d'une grandeur sensorielle, on considère que seule une définition sensorielle est apte à résoudre le problème. Diverses variantes existent concernant la méthode de rapprochement sensations - terme de texture, comme la dégustation préliminaire de produits prédéfinis (Murray et Delahunty 1999) ou l'analyse statistique multidimensionnelle de résultats; mais, en tout état de cause, la définition donnée au terme reste toujours très volatile, et une redéfinition sensorielle de la terminologie utilisée est nécessaire à chaque test entrepris. Donc, si ces méthodes peuvent présenter une grande utilité locale, au sens unité de lieu et de temps, elles sont par contre sans intérêt réel du point de vue de mise au point d'une terminologie largement acceptée par le milieu scientifique concerné. Pourtant, comme le rappelle Zannoni (1997) : «The most serious problem facing the international sensory evaluation community is the language barrier.»

Une seconde solution basée sur les impressions sensorielles a été efficacement utilisée dans la dégustation du vin, et pourrait être adaptée à l'analyse de texture des aliments. Le vocabulaire utilisé en œnologie est issu de la langue naturelle et même de la culture propre aux amateurs de vin, mais les termes ont été précisément redéfinis: «Une jolie chair en bouche, de bons tannins, une très belle expression aromatique et une bonne persistance. L'équilibre entre structure et charnu est bien réussi, la matière est belle. C'est un vin riche, gras, chaud...» (Coutier 1994).

Cette évolution de certains termes du langage naturel vers un langage spécialisé s'est réalisé grâce aux nombreux œnologues et œnophiles qui ont précisé le vocabulaire de dégustation et en ont tiré des glossaires spécialisés. Ce travail a demandé des 
dizaines d'années mais a conduit à une terminologie particulièrement originale et efficace. Théoriquement, une telle évolution dans le domaine de la texture n'est pas impossible. Mais le caractère plus mécanique qu'hédonique du domaine, marqué notamment par l'orientation instrumentale, de plus en plus marquée, de l'évaluation de la texture, ne favorise pas cette évolution.

\section{Les méthodes à base instrumentale}

L'analyse de texture instrumentale propose des méthodes très proches de la rhéologie des solides, c'est-à-dire que les principaux tests correspondent à des écrasements, torsions, percements, extensions, etc. du produit testé. Dès lors, assez naturellement, ses praticiens se sont tournés vers la terminologie physique existante dans ce domaine, qui est très éloignée de la terminologie en analyse sensorielle: on parle alors de caractéristiques mécaniques telles que coefficient d'élasticité, contrainte limite en compression, module d'Young (GFR 1990), et plus de fermeté, gluant ou croquant. Cette nouvelle terminologie a l'avantage d'être parfaitement et précisément définie, ce qui la rend apte à la communication scientifique. Cependant, très rapidement deux problèmes apparaissent: le premier concerne l'impossibilité de traduire les termes sensoriels en termes rhéologiques, et réciproquement, ce qui ne fait qu'accroître la cacophonie existante dans ce domaine. Le second problème a sa source dans la sémantique des termes mécaniques. En effet, ceux-ci ont été créés pour être utilisés pour des matériaux dont la structure n'a que peu de rapport avec celle des aliments, et si l'on peut exercer les mêmes tests dans les deux cas, il y a fort peu de chances que le paramètre mesuré ait la même signification physique. Ainsi, on attribue arbitrairement un nom à une caractéristique qui n'a, peut-être, qu'un très lointain rapport avec la caractéristique portant le même nom dans le domaine des matériaux. En poussant un peu plus loin le raisonnement, on aboutit à la conclusion que deux produits alimentaires différents pourraient posséder chacun une caractéristique différente possédant le même nom. L'intérêt de cette terminologie mécanique serait alors complètement annihilé. Heureusement, on cherche, en général, à comparer des produits similaires et ce dernier point ne pose pas de réel problème pratique.

À l'heure actuelle, la situation terminologique en est à ce stade où les professionnels de l'analyse sensorielle utilisent leur vocabulaire classique, alors que les adeptes de l'analyse instrumentale essaient de jongler avec les deux vocabulaires, sensoriel et rhéologique. Ce passage d'un vocabulaire à l'autre a le mérite de permettre l'expression de caractéristiques complexes purement texturales et donc non aisément exprimables en langage rhéologique. Cependant, cette solution n'apporte aucune réponse aux communications extra-laboratoires (changement d'expérimentateurs) ou internationales (traduction), et ne lève pas l'ambiguïté de certains termes communs aux deux lexiques, tels que la dureté par exemple qui est physiquement liée à la difficulté de déformation et sensoriellement liée à la difficulté de fracture.

\section{Une nouvelle terminologie basée sur la description structurale des aliments}

La situation actuelle, on l'a vu, n'est pas satisfaisante. Si l'on en croit Lavoisier, tel qu'il est cité ci-dessus, l'absence de prise en compte sérieuse de la langue à utiliser ne peut 
que provoquer la stagnation du domaine scientifique considéré. Dans la mesure où la terminologie sensorielle est, dans le meilleur des cas, insuffisante et où la terminologie de la rhéologie des matériaux est globalement inadaptée, il convient de proposer une nouvelle voie. Plusieurs chercheurs ont alors proposé de reprendre le travail à la base, en considérant que les termes à utiliser doivent être liés non plus aux sensations ressenties mais aux modifications de structure des produits: la texture provenant dans un premier temps de la structure physique de l'aliment, et, seulement dans un deuxième temps, de la sensation ressentie lors de la modification de cette structure. Ainsi, Civille et Lawless (1986) considèrent que les conditions auxquelles doivent répondre les termes d'un nouveau glossaire sont les suivantes:

1- Ils doivent être orthogonaux (c'est-à-dire non corrélés entre eux).

2- Ils doivent être basés sur la structure sous jacente du produit lorsqu'elle est connue.

3- Ils doivent être validés sur un grand nombre d'échantillons.

4- Ils doivent être définis avec précision.

Cette proposition trouve une bonne adhésion dans la communauté des instrumentalistes. Ainsi, Peleg (1983) va même plus loin en précisant le deuxième point, puisqu'il pense que si la modification de structure n'est pas connue, il est nécessaire de procéder à des tests complémentaires pour l'évaluer avant d'appliquer un terme de texture particulier. Cette méthode permettrait alors de différencier les notions de wet et $d r y$-crisp vues plus haut, mais aussi de différencier le terme 'juteux' utilisé pour la viande ou les agrumes, ou d'améliorer la compréhension de la notion couverte par le terme 'fermeté', très utilisé en sensoriel et uniformément rejeté par les instrumentalistes, car recouvrant des notions trop floues. Cette nouvelle approche ne consiste cependant pas à rejeter la culture sensorielle, mais à reconsidérer le problème du choix terminologique en le réorientant vers le produit et sa structure physicochimique plutôt que vers la sensation ressentie lors de la mise en bouche, comme actuellement. L'utilisation d'une telle terminologie dans le domaine sensoriel ne devrait pas modifier en profondeur les habitudes des dégustateurs, mais apporter une précision qui était difficile à obtenir jusqu'à maintenant. On peut en effet prévoir que, dans la plupart des cas, les 'anciens' termes resteront mais qu'ils seront subdivisés en nouvelles catégories définies avec précision, généralement atteignables uniquement par des méthodes instrumentales fines. Cette nouvelle terminologie correspondrait alors à un affinement de la terminologie sensorielle actuelle. On aura alors affaire à un type de taxinomie un peu dégradé car le flou de la terminologie sensorielle sera conservé pour la facilité des analyses de dégustation. Par exemple, un premier niveau de texture pourra correspondre au terme fragile, suivi au deuxième niveau des termes de croustillant, croquant et craquant (tous ces termes correspondant à la terminologie et à la hiérarchie actuelles) et au troisième niveau apparaîtront les nouvelles notions structurales telles que éclatement cellulaire par pression interne, éclatement cellulaire par rupture mécanique de paroi rigide, fracture intercellulaire par présence de méat, etc. Ainsi, la fermeté d'un melon sera, par exemple, exprimée instrumentalement par 'fermeté par élasticité élevée de la peau', alors que la fermeté d'un fromage deviendra 'fermeté par viscosité élevée'.

Une telle évolution est souhaitable tant sur le plan des concepts utilisés dans ce domaine scientifique, car cela permettrait enfin de savoir précisément à quoi on se réfère, et ainsi ne plus considérer le terme comme plus important que la texture 
elle-même, que sur le plan de la traduction interlangue, car la terminologie purement sensorielle serait alors systématiquement associée à une terminologie précisément définie. Cependant, une telle évolution n'est envisageable qu'à la suite d'importants progrès dans le domaine de la connaissance de la physique des milieux biologiques, et donc de la mise au point de théories mécaniques de ces milieux (Booth et al., 2003; Roudot, 2004). Et l'on en revient ainsi à Lavoisier: les développements scientifiques et terminologiques vont de pair.

\section{RÉFÉRENCES}

Andani, Z., JAeger, S.R., Wakeling, I. et H.J.H. Macfie (2001): "Mealiness in Apples: Towards a multilingual consumer vocabulary", Journal of Food Science 66-6, pp. 872-879.

BAGOT, J.D. (1999): Information, sensation et perception, Paris, Armand Colin.

Ball, C.O., Clauss, H.E. and E.F. STIEFer (1957): "Factors Affecting Quality of Prepackaged Meat. Loss of Weight and Study of Texture", Food Technology 11, p. 281.

Barthes, R. (1970): «Pour une psycho-sociologie de l'alimentation contemporaine», Cahiers des Annales 28, p. 307-315.

Bérodier, F., Lavanchy, P., Zannoni, M., Casals, J., Herrero, L. et C. Adamo (1997): «Guide d'évaluation olfacto-gustative des fromages à pâte dure et semi-dure», Lebensmittel-Wissenschaft und Technologie 30, pp. 653-664.

Booth, D.A., EarL, T. and S. Mobini (2003): "Perceptual channels for the texture of a food", Appetite 40, pp. 69-76.

Civille, G.V.and H.T. Lawless (1986) : "The Importance of Language in Describing Perceptions", Journal of Sensory Studies 1, pp. 203-215.

Coutier, M. (1994): «Tropes et termes: le vocabulaire de la dégustation du vin», Meta 39-4, pp. 662-675.

DANiel, C. (2003): Étude lexicographique et terminologique de la texture 'crisp' des aliments et application à la traduction Anglais-Français, Mémoire de DESS en Rédaction/Traduction, Université de Bretagne Occidentale, Brest, France.

Daniel, C. et A.-C. Roudot (2003): “On Food Texture Terminology”, Jubilee Scientific Conference HIFFI, Plovdiv (Bulgarie), in Scientific Works, University of Food Technologies, Plovdiv, p. 7-11.

Deletre, K. et A.-C. Roudot (2003): «Terminologie comparée français-anglais en analyse de texture des aliments", Sciences des Aliments 23-3, pp. 463-480.

Drake, B. (1989): "Sensory Textural/Rheological Properties - A Polyglot List", Journal of Texture Studies 20, pp. 1-27.

Eco, U. (1997): La recherche de la langue parfaite dans la culture européenne, Paris, Le Seuil.

Fillion, L. and D. KilCAst (2002): "Consumer Perception of Crispness and Crunchiness in Fruits and Vegetables", Food Quality and Preference 13, pp. 23-29.

GFR (1990): Dictionnaire de rhéologie, Français - Anglais - Allemand - Espagnol, Groupe Français de Rhéologie, Institut de Mécanique des Fluides, Toulouse, France.

HaLL, R.C. and H.C. Fryer (1953): "Consistency Evaluation of Dehydrated Potato Granules and Direction for Microscopic Rupture Count Procedure”, Food Technology 7, p. 373.

JowitT, R. (1974): "The terminology of food texture", Journal of Texture Studies 5, pp. 351-358.

Lawless, H., Vanne, M. et H. Tuorila (1997): "Categorization of English and Finnish Texture Terms Among Consumers and Food Professionals", Journal of Texture Studies 28, pp. 687708.

Mellor, S.J. (2002): Termes et qualificatifs de la dégustation, Mémoire de DESS, Université Sorbonne, Paris 3.

Murray, J.M. et C.M. Delahunty (1999): "Assessing the Value of an Agreed Vocabulary to Describe the Image for Retail of Cheddar-Type Cheese", in Proceedings of the $10^{\text {th }}$ World Congress of Food Science and Technology, Sydney, Australia. 
Peleg, M. (1983): “The Semantics of Rheology and Texture”, Food Technology 11, pp. 54-61.

Rodriguez, J.L., Rodriguez, I. et R. De Hombre (1997): “Terminos empleados en la evaluacion sensorial de la textura de alimentos", Alimentaria 35-283, pp. 83-86.

Roudot, A-C. (2004): “Terminology in Food Quality Management", $5^{\text {th }}$ International Conference on Quality - Reliability - Maintenance, in McNulty, G.J. (ed.), Professional Engineering Publishing Limited, Bury St Edmunds, pp. 39-42.

Sarrukai, S. (2001a): "Translation and Science", Meta 56-4, pp. 646-663.

Sarrukai, S. (2001b): "Mathematics, Language and Translation", Meta 56-4, pp. 664-674.

Sмith, H.R. (1947): "Objective Measurement of Quality in Foods", Food Technology 1, pp. 345.

Szczesniak, A.S. (1963) : “Classification of Textural Characteristics”, Journal of Food Science 28-4, pp. 385-389.

Szczesniak, A.S. (1988): “The Meaning of Textural Characteristics - Crispness", Journal of Texture Studies 19, pp. 51-59.

Szczesniak, A.S. (1998): "Sensory Texture Profiling - Historical and Scientific Perspectives", Food Technology 52-8, pp. 54-57.

SzCZesniak, A.S. and E.S. Skinner (1973): "Meaning of Texture Words to the Consumer", Journal of. Texture Studies 4, pp. 378-384.

This, J. (2002): Traité élémentaire de cuisine, Paris, Belin.

Zannoni, M. (1997): "Approaches to Translation Problems of Sensory Descriptors", Journal of Sensory Studies 12, pp. 239-253. 